\title{
ACCURATE SOLUTIONS OF SCATTERING PROBLEMS INVOLVING LOW-CONTRAST DIELECTRIC OBJECTS WITH SURFACE INTEGRAL EQUATIONS
}

\author{
Ö. Ergüll ${ }^{1,2}$, L. Gürel ${ }^{1,2}$ \\ ${ }^{1}$ Department of Electrical and Electronics Engineering \\ ${ }^{2}$ Computational Electromagnetics Research Center (BiLCEM) \\ Bilkent University, TR-06800, Bilkent, Ankara, Turkey \\ E-mail: ergul@ee.bilkent.edu.tr, lgurel@bilkent.edu.tr \\ fax: +90-312-2905755
}

Keywords: Electromagnetic scattering, dielectrics, surface integral equations, multilevel fast multipole algorithm.

\begin{abstract}
We present the stabilization of the surface integral equations for accurate solutions of scattering problems involving low-contrast dielectric objects. Unlike volume formulations, conventional surface formulations fail to provide accurate results for the scattered fields when the contrast of the object is small. Therefore, surface formulations are required to be stabilized by extracting the nonradiating parts of the equivalent currents. In addition to previous strategies for the stabilization, we introduce a novel procedure called field-based stabilization (FBS) based on using fictitious incident fields and rearranging the right-hand-side of the equations. The results show that the formulations using FBS provide accurate results even for scattering problems involving extremely low-contrast objects, while the extra cost due to the stabilization procedure is negligible.
\end{abstract}

\section{Introduction}

Surface integral equations are commonly used to formulate scattering problems involving homogenous dielectric targets with arbitrary shapes and electrical properties [7]. Depending on the choices of the strategies for combining the boundary conditions, normalizing the fields, and choosing a testing scheme, there are many formulations that are free of the internal resonance problem and provide stable solutions [6][7],[9]-[12]. On the other hand, surface formulations fail to provide accurate results for the scattered fields (as opposed to volume formulations) when the contrast of the object is small, i.e., when the electromagnetic material properties of the target and the host medium are close to each other [4]. This inaccuracy is due to the large nonradiating components of the equivalent electric and magnetic currents, which dominate the radiating currents as the contrast decreases.
In other words, even by using a fine discretization for the object and the integral equations, the important part of the surface currents cannot be modelled accurately when the object is less visible and the scattered fields become low. As a remedy, a stabilization method, which is based on extracting the nonradiating parts of the currents from the total currents, can be used [4]. Recently, we showed that such a stabilization procedure can be applied to various formulations by splitting the currents into radiating and nonradiating parts, applying the integral operators on the nonradiating parts, rearranging the right-hand side (RHS) of the matrix equations, and solving only for the radiating currents [2]. However, this procedure, which we call operator-based stabilization (OBS), is successful only when the contrast of the object is not very low (usually larger than $0.01 \%$ ). In addition, the accuracy of the results can be sensitive to the accuracy of the discretization of the operators, which is undesirable especially when the matrix-vector multiplications are performed approximately by accelerated methods, such as the fast multipole method (FMM) and the multilevel fast multipole algorithm (MLFMA) [9].

In OBS, the RHSs of the matrix equations involve subtraction of the inner and outer operators from each other. This leads to vanishing RHSs as the contrast goes down and provides an accurate calculation of the radiating currents for moderately low contrasts. In this paper, we present a novel stabilization procedure, which is based on subtracting the fields from each other; this procedure does not suffer from a breakdown. In this strategy, which we call the field-based stabilization (FBS), we again extract the nonradiating parts of the currents and model only the radiating currents. On the other hand, the RHSs of the equations are arranged in a different manner based on using fictitious incident fields defined by exchanging the electromagnetic material properties of the inner and outer media. Then, the RHS involves subtraction operation for the fields instead of the operators. This way, the stabilization becomes more robust 
and less sensitive to the accuracy of the matrix-vector multiplications. In our experiments, we show that FBS is accurate for the solution of scattering problems involving arbitrarily low-contrast objects, even when the contrast is extremely low (for example, $0.0000001 \%$ ). In this paper, we compare the conventional and the stabilized formulations on scattering problems involving sphere geometries with various sizes and contrasts to demonstrate the robustness of FBS.

\section{Surface Formulations of Dielectric Problems}

In the surface formulations of scattering problems involving homogenous dielectric objects, equivalent electric and magnetic currents are defined on the surface of the scatterer, i.e.,

$$
\boldsymbol{J}(\boldsymbol{r})=\hat{\boldsymbol{n}} \times \boldsymbol{H}(\boldsymbol{r}) \quad \boldsymbol{M}(\boldsymbol{r})=-\hat{\boldsymbol{n}} \times \boldsymbol{E}(\boldsymbol{r}) .
$$

Then, the boundary conditions are tested on the surface to calculate the unknown surface currents and the scattered fields. In the literature, there are various integral-equation formulations derived by using different combinations of the boundary conditions and testing schemes [6]-[7],[9]-[12]. Let the exterior and the interior of the object with surface $S$ be characterized by electromagnetic parameters $\left(\epsilon_{1}, \mu_{1}\right)$ and $\left(\epsilon_{2}, \mu_{2}\right)$, respectively. When the tangential components of the fields are directly sampled (tested) on the surface, the $\mathrm{T}$ formulations are derived as [11]

$$
\hat{\boldsymbol{t}} \cdot\left[\begin{array}{cc}
\mathcal{Z}_{11}^{T} & \mathcal{Z}_{12}^{T} \\
\mathcal{Z}_{21}^{T} & \mathcal{Z}_{22}^{T}
\end{array}\right] \cdot\left[\begin{array}{c}
\boldsymbol{J}(\boldsymbol{r}) \\
\boldsymbol{M}(\boldsymbol{r})
\end{array}\right]=-\hat{\boldsymbol{t}} \cdot\left[\begin{array}{c}
a \eta_{1}^{-1} \boldsymbol{E}^{i}(\boldsymbol{r}) \\
c \eta_{1} \boldsymbol{H}^{i}(\boldsymbol{r})
\end{array}\right]
$$

where

$$
\begin{aligned}
& \mathcal{Z}_{11}^{T}=a \mathcal{T}_{1}+b \mathcal{T}_{2} \\
& \mathcal{Z}_{12}^{T}=-a \eta_{1}^{-1} \mathcal{K}_{1}-b \eta_{2}^{-1} \mathcal{K}_{2}+0.5\left(b \eta_{2}^{-1}-a \eta_{1}^{-1}\right) \hat{\boldsymbol{n}} \times \mathcal{I} \\
& \mathcal{Z}_{21}^{T}=c \eta_{1} \mathcal{K}_{1}+d \eta_{2} \mathcal{K}_{2}+0.5\left(c \eta_{1}-d \eta_{2}\right) \hat{\boldsymbol{n}} \times \mathcal{I} \\
& \mathcal{Z}_{22}^{T}=c \mathcal{T}_{1}+d \mathcal{T}_{2}
\end{aligned}
$$

and $\{a, b, c, d\}$ represent the combination parameters. In (2)(6), $\boldsymbol{E}^{i}(\boldsymbol{r})$ and $\boldsymbol{H}^{i}(\boldsymbol{r})$ are the incident electric and magnetic fields, $\eta_{l}=\sqrt{\mu_{l} / \epsilon_{l}}$ is the impedance of the medium $l=1,2$, $\hat{\boldsymbol{t}}$ is any tangential vector on the surface, and $\hat{\boldsymbol{n}}$ is the outward normal vector. In addition, the operators are defined as

$$
\mathcal{T}_{l}\{\boldsymbol{X}(\boldsymbol{r})\}=i k_{l} \int_{S} d \boldsymbol{r}^{\prime}\left[\boldsymbol{X}\left(\boldsymbol{r}^{\prime}\right)+\frac{1}{k_{l}^{2}} \nabla \nabla^{\prime} \cdot \boldsymbol{X}\left(\boldsymbol{r}^{\prime}\right)\right] g_{l}\left(\boldsymbol{r}, \boldsymbol{r}^{\prime}\right)
$$

$$
\begin{aligned}
\mathcal{K}_{l}\{\boldsymbol{X}(\boldsymbol{r})\} & =\int_{P V, S} d \boldsymbol{r}^{\prime} \boldsymbol{X}\left(\boldsymbol{r}^{\prime}\right) \times \nabla^{\prime} g_{l}\left(\boldsymbol{r}, \boldsymbol{r}^{\prime}\right) \\
\mathcal{I}\{\boldsymbol{X}(\boldsymbol{r})\} & =\boldsymbol{X}(\boldsymbol{r}),
\end{aligned}
$$

where $P V$ indicates the principal value of the integral, $k_{l}=$ $w \sqrt{\epsilon_{l} \mu_{l}}$ is the wavenumber associated with medium $l$, and $g_{l}\left(\boldsymbol{r}, \boldsymbol{r}^{\prime}\right)$ denotes the homogeneous-space Green's function defined as

$$
g_{l}\left(\boldsymbol{r}, \boldsymbol{r}^{\prime}\right)=\frac{\exp \left(i k_{l} R\right)}{4 \pi R} \quad\left(R=\left|\boldsymbol{r}-\boldsymbol{r}^{\prime}\right|\right)
$$

in phasor notation using the $\exp (-i w t)$ convention. Among various $\mathrm{T}$ formulations, the tangential Poggio-Miller-ChangHarrington-Wu-Tsai (T-PMCHWT) [7] formulation and the combined $\mathrm{T}$ formulation (CTF) [11] are well known and commonly used in the literature. Both of these formulations are free of the internal-resonance problem.

Unlike the $\mathrm{T}$ formulations, the $\mathrm{N}$ formulations are written as [11]

$$
\hat{\boldsymbol{n}} \times\left[\begin{array}{cc}
\mathcal{Z}_{11}^{N} & \mathcal{Z}_{12}^{N} \\
\mathcal{Z}_{21}^{N} & \mathcal{Z}_{22}^{N}
\end{array}\right] \cdot\left[\begin{array}{c}
\boldsymbol{J}(\boldsymbol{r}) \\
\boldsymbol{M}(\boldsymbol{r})
\end{array}\right]=-\hat{\boldsymbol{n}} \times\left[\begin{array}{c}
a \boldsymbol{H}^{i}(\boldsymbol{r}) \\
-c \boldsymbol{E}^{i}(\boldsymbol{r})
\end{array}\right]
$$

by using a cross product with the outward normal vector to obtain the tangential field components on the surface of the scatterer. The operators in (11) are defined as

$$
\begin{aligned}
& \mathcal{Z}_{11}^{N}=a \mathcal{K}_{1}-b \mathcal{K}_{2}+0.5(a+b) \hat{\boldsymbol{n}} \times \mathcal{I} \\
& \mathcal{Z}_{12}^{N}=a \eta_{1}^{-1} \mathcal{T}_{1}-b \eta_{2}^{-1} \mathcal{T}_{2} \\
& \mathcal{Z}_{21}^{N}=-c \eta_{1} \mathcal{T}_{1}+d \eta_{2} \mathcal{I}_{2} \\
& \mathcal{Z}_{22}^{N}=c \mathcal{K}_{1}-d \mathcal{K}_{2}+0.5(c+d) \hat{\boldsymbol{n}} \times \mathcal{I} .
\end{aligned}
$$

Among various $\mathrm{N}$ formulations, the combined $\mathrm{N}$ formulation (CNF) [11] and the $\mathrm{N}$ Müller formulation (NMF) [10] are free of the internal-resonance problem and extensively used to formulate the scattering problems of dielectric objects.

\section{Low-Contrast Breakdown}

For the solution of scattering problems involving dielectric objects with moderate contrasts, both $\mathrm{T}$ and $\mathrm{N}$ formulations can be used successfully. Due to well-tested identity terms, $\mathrm{N}$ formulations are usually better-conditioned than the $\mathrm{T}$ formulations, which contain weakly-tested identity terms [11]. Therefore, iterative solutions of the $\mathrm{N}$ formulations are easier, which is essential especially when the problem size is large. On the other hand, $\mathrm{N}$ formulations can be inaccurate compared to the $\mathrm{T}$ formulations for the same discretization; this is also due to the well-tested identity terms [1],[11]. Consequently, the choice of the formulation for the solution of a problem depends on the accuracy and the efficiency requirements.

Although they are accurate to formulate scattering problems with moderate contrasts, conventional surface integral equations become inaccurate to calculate the scattered fields when the contrast of the dielectric object decreases, i.e., when the electromagnetic material properties of the target and the host medium are close to each other. This breakdown is due to the large nonradiating parts of the induced currents on the scatterer [4]. For any arbitrary solution, equivalent electric and magnetic currents on the surface can be decomposed as

$$
\begin{aligned}
\boldsymbol{J}(\boldsymbol{r}) & =\hat{\boldsymbol{n}} \times \boldsymbol{H}(\boldsymbol{r})=\hat{\boldsymbol{n}} \times \boldsymbol{H}^{i}(\boldsymbol{r})+\hat{\boldsymbol{n}} \times \boldsymbol{H}^{r}(\boldsymbol{r}) \\
\boldsymbol{M}(\boldsymbol{r}) & =-\hat{\boldsymbol{n}} \times \boldsymbol{E}(\boldsymbol{r})=-\hat{\boldsymbol{n}} \times \boldsymbol{E}^{i}(\boldsymbol{r})-\hat{\boldsymbol{n}} \times \boldsymbol{E}^{r}(\boldsymbol{r}),
\end{aligned}
$$

where $\hat{\boldsymbol{n}} \times \boldsymbol{H}^{i}(\boldsymbol{r})$ and $\hat{\boldsymbol{n}} \times \boldsymbol{E}^{i}(\boldsymbol{r})$ do not radiate. When the contrast of the object decreases, i.e., when $\epsilon_{2} \rightarrow \epsilon_{1}$ and 


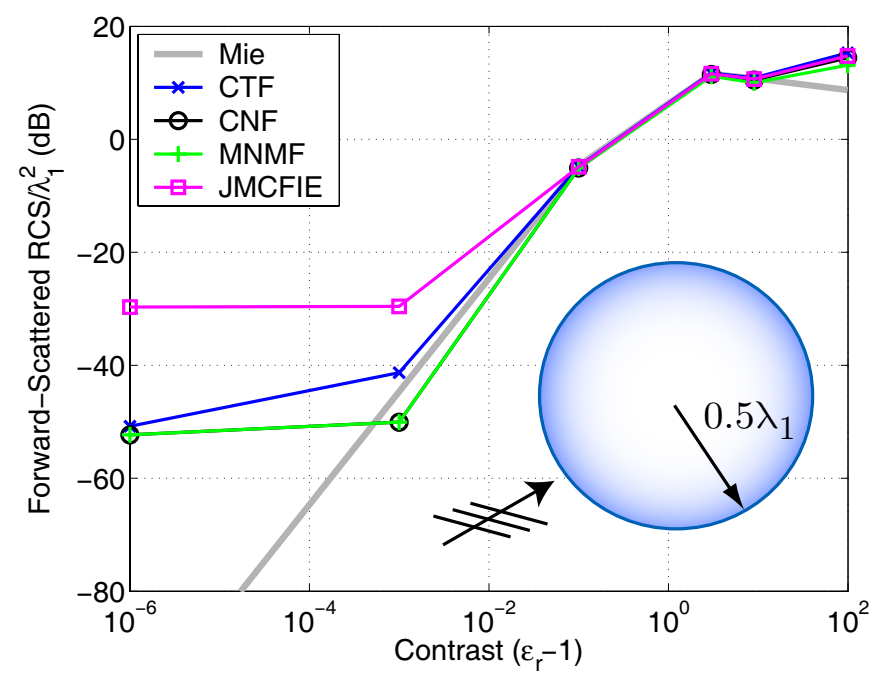

Fig. 1: Normalized forward-scattered RCS $\left(\operatorname{RCS} / \lambda_{1}^{2}\right)$ of a sphere of radius $0.5 \lambda_{1}$ as a function of contrast $\left(\epsilon_{r}-1\right)$. Computational values are obtained with $\lambda_{1} / 10$ triangulation.

$\mu_{2} \rightarrow \mu_{1}$, these nonradiating currents dominate the total currents. Therefore, it becomes difficult to correctly model the small radiating currents, i.e., $\hat{\boldsymbol{n}} \times \boldsymbol{H}^{r}(\boldsymbol{r})$ and $\hat{\boldsymbol{n}} \times \boldsymbol{E}^{r}(\boldsymbol{r})$. In other words, when the total currents are solved by employing the conventional surface formulations, it is difficult to perform the calculations sensitive enough to model the small radiating currents properly. Although the total surface currents are calculated accurately, scattered fields cannot be obtained from them with the desired level of accuracy.

As an example, Fig. 1 presents the results of scattering problems involving a sphere of radius $0.5 \lambda_{1}$, where $\lambda_{1}$ is the wavelength outside the sphere. The sphere is inside the free space and we consider various relative dielectric constants $\left(\epsilon_{r}\right)$ for the sphere from 1.000001 to 100 . In Fig. 1, normalized forward-scattered radar cross section values (RCS $/ \lambda_{1}^{2}$ in $\mathrm{dB}$ ) are plotted as a function of the contrast defined as $\epsilon_{r}-1$. To apply different formulations, the sphere is discretized by using planar triangles with a mesh size of $\lambda_{1} / 10$, on which RaoWilton-Glisson (RWG) [8] functions are defined, leading to 1860 unknowns. We check the accuracy of the formulations by comparing the computational RCS values with the analytical results obtained by Mie-series solutions. Among various formulations, we consider $\mathrm{CTF}, \mathrm{CNF}$, a modified (improved) NMF (MNMF) [10], and the electric-magnetic current combined-field integral equation (JMCFIE) [12] obtained by combining CTF and CNF. Fig. 1 shows that all formulations provide accurate results for moderate contrasts, while they become inaccurate for low and high contrasts. The accuracy problem in the high-contrast solutions is due to the insufficient modelling of the geometries by planar triangles and it can be avoided easily by improving the discretization of the geometry using either a finer triangulation or curved elements. On the other hand, the "breakdown" in the lowcontrast solutions is due to the large non-radiating parts of the equivalent currents on the surface. Since the error for the low-contrast solutions cannot be eliminated easily with better discretizations, we need to stabilize the integral equations.

\section{Stabilization of Surface Integral Equations}

For accurate solutions of low-contrast problems, dielectric formulations should be modified by extracting the incident fields from the total currents and modelling only the radiating currents as the unknowns [4]. In this paper, we consider the stabilization of a modified CTF and compare the results of the conventional and the stabilized implementations. Originally, CTF is derived in [11] by choosing $\{a, b, c, d\}=\{1,1,1,1\}$ in (2)-(6). However, we take $\{a, b, c, d\}=\left\{\eta_{1}, \eta_{2}, \eta_{2}, \eta_{1}\right\}$, which results in a similar formulation compared to the original $\mathrm{CTF}$ for low-contrast problems $\left(\eta_{2} \approx \eta_{1}\right)$. Вy modifying the combination parameters as above, we eliminate the weakly-tested identity terms from the equation and obtain a simpler formulation to apply the stabilizations. Furthermore, the stabilization procedures that are considered in this paper can easily be applied to other $\mathrm{T}$ and $\mathrm{N}$ formulations.

\subsection{Operator-Based Stabilization}

$$
\begin{aligned}
& \text { Choosing }\{a, b, c, d\}=\left\{\eta_{1}, \eta_{2}, \eta_{2}, \eta_{1}\right\} \text { in (2)-(6), } \\
& \mathcal{Z}_{11}^{T}=\eta_{1} \mathcal{T}_{1}+\eta_{2} \mathcal{T}_{2} \\
& \mathcal{Z}_{12}^{T}=-\mathcal{K}_{1}-\mathcal{K}_{2} \\
& \mathcal{Z}_{21}^{T}=\eta_{1} \eta_{2} \mathcal{K}_{1}+\eta_{1} \eta_{2} \mathcal{K}_{2} \\
& \mathcal{Z}_{22}^{T}=\eta_{2} \mathcal{T}_{1}+\eta_{1} \mathcal{T}_{2}
\end{aligned}
$$

Extracting the nonradiating parts of the currents and using the identity [5]

$$
\hat{\boldsymbol{t}} \cdot\left[\begin{array}{cc}
\eta_{1} \mathcal{T}_{1} & -\mathcal{K}_{1} \\
\mathcal{K}_{1} & \eta_{1}^{-1} \mathcal{T}_{1}
\end{array}\right] \cdot\left[\begin{array}{c}
-2 \hat{\boldsymbol{n}} \times \boldsymbol{H}^{i}(\boldsymbol{r}) \\
2 \hat{\boldsymbol{n}} \times \boldsymbol{E}^{i}(\boldsymbol{r})
\end{array}\right]=\hat{\boldsymbol{t}} \cdot\left[\begin{array}{c}
\boldsymbol{E}^{i}(\boldsymbol{r}) \\
\boldsymbol{H}^{i}(\boldsymbol{r})
\end{array}\right],
$$

we obtain

$$
\begin{aligned}
\hat{\boldsymbol{t}} \cdot\left[\begin{array}{cc}
\mathcal{Z}_{11}^{T} & \mathcal{Z}_{12}^{T} \\
\mathcal{Z}_{21}^{T} & \mathcal{Z}_{22}^{T}
\end{array}\right] \cdot\left[\begin{array}{c}
\hat{\boldsymbol{n}} \times \boldsymbol{H}^{r}(\boldsymbol{r}) \\
-\hat{\boldsymbol{n}} \times \boldsymbol{E}^{r}(\boldsymbol{r})
\end{array}\right] \\
=\hat{\boldsymbol{t}} \cdot\left[\begin{array}{cc}
\mathcal{Y}_{11}^{T} & \mathcal{Y}_{12}^{T} \\
\mathcal{Y}_{21}^{T} & \mathcal{Y}_{22}^{T}
\end{array}\right] \cdot\left[\begin{array}{c}
\hat{\boldsymbol{n}} \times \boldsymbol{H}^{i}(\boldsymbol{r}) \\
-\hat{\boldsymbol{n}} \times \boldsymbol{E}^{i}(\boldsymbol{r})
\end{array}\right],
\end{aligned}
$$

where

$$
\begin{aligned}
& \mathcal{Y}_{11}^{T}=\eta_{1} \mathcal{T}_{1}-\eta_{2} \mathcal{T}_{2} \\
& \mathcal{Y}_{12}^{T}=-\mathcal{K}_{1}+\mathcal{K}_{2} \\
& \mathcal{Y}_{21}^{T}=\eta_{1} \eta_{2} \mathcal{K}_{1}-\eta_{1} \eta_{2} \mathcal{K}_{2} \\
& \mathcal{Y}_{22}^{T}=\eta_{2} \mathcal{T}_{1}-\eta_{1} \mathcal{T}_{2} .
\end{aligned}
$$

We call (23) the operator-based-stabilized CTF (OBS-CTF), since the RHS involves the subtraction of the inner and outer operators from each other. The stabilization procedure does not modify the matrix part and it only requires extra computations involving the application of the operators on the incident fields. These computations are performed before the iterative solution of the matrix equation (obtained by 
the discretization) and the additional cost is negligible. Only one extra matrix-vector multiplication is required after the incident fields are expanded in a series of basis functions [3].

\subsection{Field-Based Stabilization}

OBS-CTF can easily be obtained from the existing implementations of CTF and it provides accurate results for the solution of low-contrast dielectric problems. On the other hand, OBS-CTF also break down and fail to provide accurate results for very low contrasts. Therefore, we need a more robust formulation that is valid for arbitrarily low contrasts. We achieve this by introducing fictitious incident fields and forming RHSs based on difference of the fields instead of the operators.

To derive the field-based-stabilized CTF (FBS-CTF), we define fictitious incident fields as

$$
\left\{\boldsymbol{E}_{2}^{i}(\boldsymbol{r}), \boldsymbol{H}_{2}^{i}(\boldsymbol{r})\right\}=\lim _{\substack{\epsilon_{1} \rightarrow \epsilon_{2} \\ \mu_{1} \rightarrow \mu_{2}}}\left\{\boldsymbol{E}^{i}(\boldsymbol{r}), \boldsymbol{H}^{i}(\boldsymbol{r})\right\}
$$

by using the electromagnetic material properties of the inner medium for the outside. Then, similar to the identity in (22), we have

$\hat{\boldsymbol{t}} \cdot\left[\begin{array}{cc}\eta_{2} \mathcal{T}_{2} & -\mathcal{K}_{2} \\ \mathcal{K}_{2} & \eta_{2}^{-1} \mathcal{T}_{2}\end{array}\right] \cdot\left[\begin{array}{c}-2 \hat{\boldsymbol{n}} \times \boldsymbol{H}_{2}^{i}(\boldsymbol{r}) \\ 2 \hat{\boldsymbol{n}} \times \boldsymbol{E}_{2}^{i}(\boldsymbol{r})\end{array}\right]=\hat{\boldsymbol{t}} \cdot\left[\begin{array}{c}\boldsymbol{E}_{2}^{i}(\boldsymbol{r}) \\ \boldsymbol{H}_{2}^{i}(\boldsymbol{r})\end{array}\right]$

Finally, using (29) in (23) and rearranging the equation, we obtain FBS-CTF as

$$
\begin{aligned}
& \hat{\boldsymbol{t}} \cdot\left[\begin{array}{cc}
\mathcal{Z}_{11}^{T} & \mathcal{Z}_{12}^{T} \\
\mathcal{Z}_{21}^{T} & \mathcal{Z}_{22}^{T}
\end{array}\right] \cdot\left[\begin{array}{c}
\hat{\boldsymbol{n}} \times \boldsymbol{H}^{r}(\boldsymbol{r}) \\
-\hat{\boldsymbol{n}} \times \boldsymbol{E}^{r}(\boldsymbol{r})
\end{array}\right] \\
= & -0.5 \hat{\boldsymbol{t}} \cdot\left[\begin{array}{c}
\boldsymbol{E}^{i}(\boldsymbol{r})-\boldsymbol{E}_{2}^{i}(\boldsymbol{r}) \\
\eta_{1} \eta_{2}\left(\boldsymbol{H}^{i}(\boldsymbol{r})-\boldsymbol{H}_{2}^{i}(\boldsymbol{r})\right)
\end{array}\right] \\
- & \hat{\boldsymbol{t}} \cdot\left[\begin{array}{cc}
\eta_{2} \mathcal{T}_{2} & -\mathcal{K}_{2} \\
-\eta_{1} \eta_{2} \mathcal{K}_{2} & \eta_{2} \mathcal{T}_{2}
\end{array}\right] \cdot\left[\begin{array}{c}
\hat{\boldsymbol{n}} \times \boldsymbol{H}^{i}(\boldsymbol{r})-\hat{\boldsymbol{n}} \times \boldsymbol{H}_{2}^{i}(\boldsymbol{r}) \\
-\hat{\boldsymbol{n}} \times \boldsymbol{E}^{i}(\boldsymbol{r})+\hat{\boldsymbol{n}} \times \boldsymbol{E}_{2}^{i}(\boldsymbol{r})
\end{array}\right] .
\end{aligned}
$$

The matrix part of the stabilized equation is again the same as the original equation. Only the RHS requires modifications involving operators applied on the incident fields. In the FBS-CTF, the real and fictitious fields are subtracted from each other, which can be performed analytically before the discretization. Then, the results of the subtraction operations are expanded in a series of basis functions to apply the inner operators on them. Similar to OBS-CTF, the additional cost compared to the original formulation is negligible. Unlike OBS-CTF, however, FBS-CTF is very robust and provides accurate results even for extremely low-contrast problems, as demonstrated in the next section.

\section{Results}

To compare the accuracy of the conventional and the stabilized formulations, Fig. 2 presents the results of scattering problems involving a sphere of radius $0.5 \lambda_{1}$ discretized with 1860 RWG functions. The sphere is inside the free space and it is

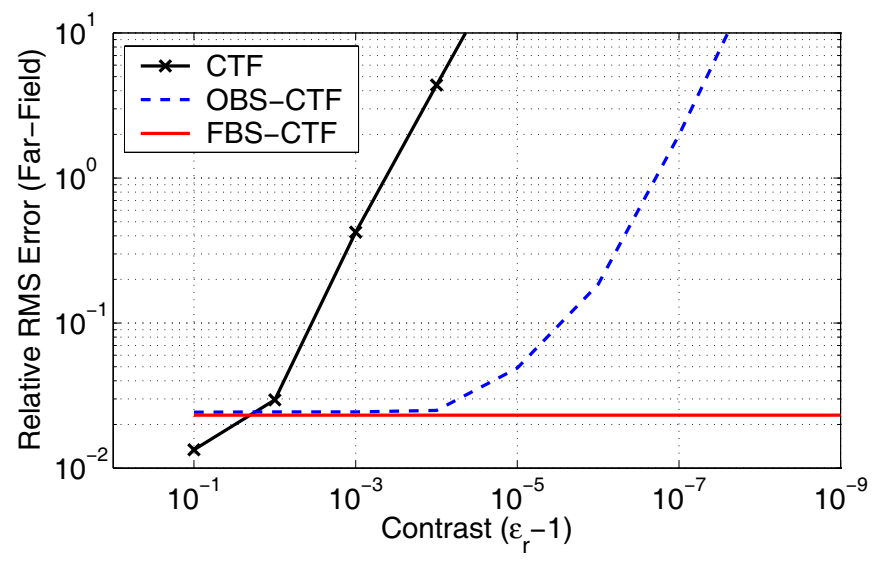

Fig. 2: Root-mean-square (RMS) error in the solution of a scattering problem involving a sphere of radius $0.5 \lambda_{1}$ in free space.

illuminated by a plane wave propagating in the $z$ direction with the electric field polarized in the $x$ direction. We consider various relative dielectric constants for the sphere from $1+10^{-9}$ to $1+10^{-1}$. Matrix equations obtained by using the conventional and the stabilized formulations are solved iteratively, where the matrix-vector multiplications are performed by using FMM. Both near-field and far-field interactions are computed with $10^{-2}$ relative error. Fig. 2 demonstrates the relative root-mean-square (RMS) error in the computed values of the far-zone electric field on the $\phi=0$ plane with respect to different contrasts $\left(\epsilon_{r}-1\right)$. We compute the far-zone electric field on the $\phi=0$ plane at 360 points from $\theta=0^{\circ}$ to $\theta=180^{\circ}$ by using the conventional CTF, OBS-CTF, and FBS-CTF. As a reference, far-zone fields are also computed analytically by the Mie-series method. Then, the RMS error is defined as

$$
e_{R M S}=\frac{\left\|\boldsymbol{f}_{C}-\boldsymbol{f}_{A}\right\|_{2}}{\left\|\boldsymbol{f}_{A}\right\|_{2}},
$$

where $\boldsymbol{f}_{C}$ and $\boldsymbol{f}_{A}$ are the computational and analytical values (complex arrays of 360 elements containing co-polar electric fields), respectively, and $\|.\|_{2}$ represents the 2-norm of the arrays.

Fig. 2 shows that the conventional CTF encounters stability problems as the contrast decreases and it fails to provide accurate results for low-contrast levels. By using OBS-CTF, however, the accuracy is stabilized in the $\left[10^{-4}, 10^{-1}\right]$ range of contrast. On the other hand, OBS-CTF also break down and its accuracy deteriorates dramatically for lower contrasts. As opposed to OBS-CTF, FBS-CTF is stable for all contrasts considered in Fig. 2 and we obtain accurate results even for $\epsilon_{r}=1+10^{-9}$. To further investigate the accuracy of the formulations, Fig. 3 depicts the bistatic RCS for three different contrasts, i.e., $10^{-1}, 10^{-5}$, and $10^{-9}$. Fig. 3(a) shows that both conventional and stabilized formulations provide accurate results that are close to the Mie-series solution when the contrast is $10^{-1}$. When the contrast is decreased to $10^{-5}$, however, conventional CTF becomes significantly 
inaccurate as shown in Fig. 3(b). Finally, Fig. 3(c) shows that only FBS-CTF provides accurate results when the contrast is extremely low $\left(10^{-9}\right)$.

Fig. 4 presents the results of a larger scattering problem involving a sphere of radius $6 \lambda_{1}$ discretized with 264,006 unknowns. Similar to the previous example, the sphere is located in free space, it is illuminated by a plane wave propagating in the $z$ direction with the electric field polarized in the $x$ direction, and we consider various relative dielectric constants for the sphere. The scattering problems are solved by MLFMA implementations of conventional and stabilized formulations. In Fig. 4, bistatic RCS is plotted for three different contrasts. The results are very similar to the previous example. We observe that the values obtained by all three formulations are close to the analytical curve when the contrast is relatively high $\left(10^{-1}\right)$. As the contrast decreases, however, the conventional CTF becomes unstable and fails to provide accurate results. Finally, only FBS-CTF is accurate when the contrast is decreased to $10^{-9}$.

\section{Conclusion}

In this paper, we report the stabilization of the surface integral equations for accurate solutions of scattering problems involving low-contrast dielectric objects. Conventional surface formulations break down when the contrast of the object is small. Consequently, scattered fields cannot be calculated accurately without a stabilization, which involves the extraction of the nonradiating currents and solving the matrix equations for relatively small radiating currents. In addition to previous strategies, we introduce a novel stabilization procedure based on using fictitious incident fields and rearranging the RHSs of the equations accordingly. We apply the stabilizations to a combined $\mathrm{T}$ formulation, although they also applicable to other $\mathrm{T}$ and $\mathrm{N}$ formulations. The stabilizations are easy to implement by modifying the existing codes for the conventional formulations and their extra computational cost is negligible. Our results show that a field-based stabilized equation, namely FBS-CTF, provides accurate results even for extremely low-contrast objects, such as a sphere with a relative electric constant of $1+10^{-9}$ in free space.

\section{Acknowledgements}

This work was supported by the Scientific and Technical Research Council of Turkey (TUBITAK) under Research Grant 105E172, by the Turkish Academy of Sciences in the framework of the Young Scientist Award Program (LG/TUBA-GEBIP/2002-1-12), and by contracts from ASELSAN and SSM.

\section{References}

[1] Ö. Ergül and L. Gürel, "Investigation of the inaccuracy of the MFIE discretized with the RWG basis functions," in Proc. IEEE Antennas and Propagation Soc. Int. Symp., vol. 3, 2004, pp. 3393-3396.

[2] Ö. Ergül and L. Gürel, "Improving the accuracy of the surface integral equations for low-contrast dielectric scatterers," in Proc. IEEE Antennas and Propagation Soc. Int. Symp., 2007, pp. 4853-4856.

[3] Ö. Ergül and L. Gürel, "Stabilization of integral-equation formulations for the accurate solution of scattering problems involving low-contrast dielectric objects," IEEE Trans. Antennas Propagat., submitted for publication.

[4] P. M. Goggans and A. Glisson, "A surface integral equation formulation for low contrast scatterers based on radiation currents," ACES Journal, vol. 10, pp. 15-18, Mar. 1995.

[5] G. C. Hsiao and R. E. Kleinman, "Mathematical foundations for error estimation in numerical solutions of integral equations in electromagnetics," IEEE Trans. Antennas Propagat., vol. 45, no. 3, pp. 316-328, Mar. 1997.

[6] T. W. Lloyd, J. M. Song, and M. Yang, "Numerical study of surface integral formulations for low-contrast objects," IEEE Antennas Wireless Propagat. Lett., vol. 4, pp. 482485, 2005.

[7] A. J. Poggio and E. K. Miller, "Integral equation solutions of three-dimensional scattering problems," in Computer Techniques for Electromagnetics, R. Mittra, Ed. Oxford: Permagon Press, 1973, Chap. 4.

[8] S. M. Rao, D. R. Wilton, and A. W. Glisson, "Electromagnetic scattering by surfaces of arbitrary shape," IEEE Trans. Antennas Propagat., vol. AP-30, no. 3, pp. 409418, May 1982.

[9] X.-Q. Sheng, J.-M. Jin, J. Song, W. C. Chew, and C.C. Lu, "Solution of combined-field integral equation using multilevel fast multipole algorithm for scattering by homogeneous bodies," IEEE Trans. Antennas Propagat., vol. 46, no. 11, pp. 1718-1726, Nov. 1998.

[10] P. Ylä-Oijala and M. Taskinen, "Well-conditioned Müller formulation for electromagnetic scattering by dielectric objects," IEEE Trans. Antennas Propagat., vol. 53, no. 10, pp. 3316-3323, Oct. 2005.

[11] P. Ylä-Oijala, M. Taskinen, and S. Järvenpää, "Surface integral equation formulations for solving electromagnetic scattering problems with iterative methods," Radio Science, vol. 40, RS6002, Nov. 2005.

[12] P. Ylä-Oijala and M. Taskinen, "Application of combined field integral equation for electromagnetic scattering by dielectric and composite objects," IEEE Trans. Antennas Propagat., vol. 53, no. 3, pp. 1168-1173, Mar. 2005. 


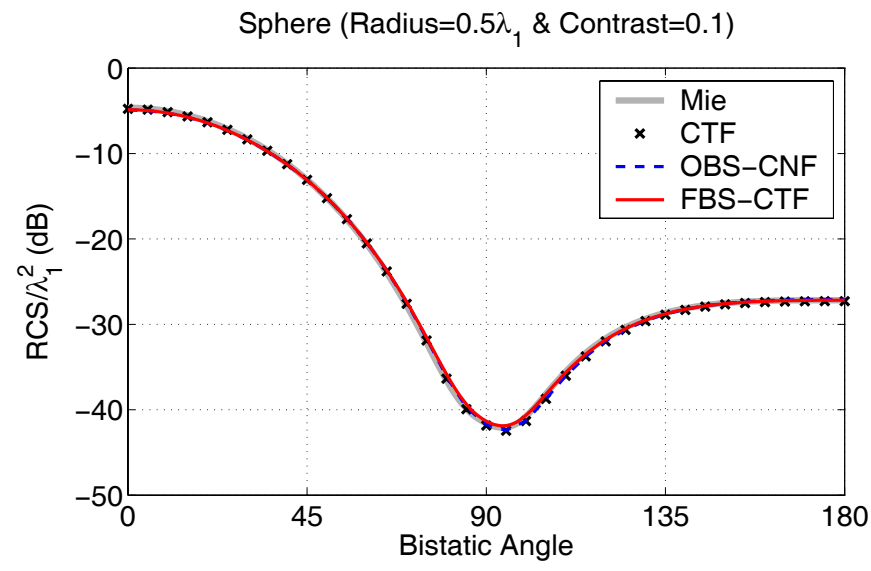

(a)

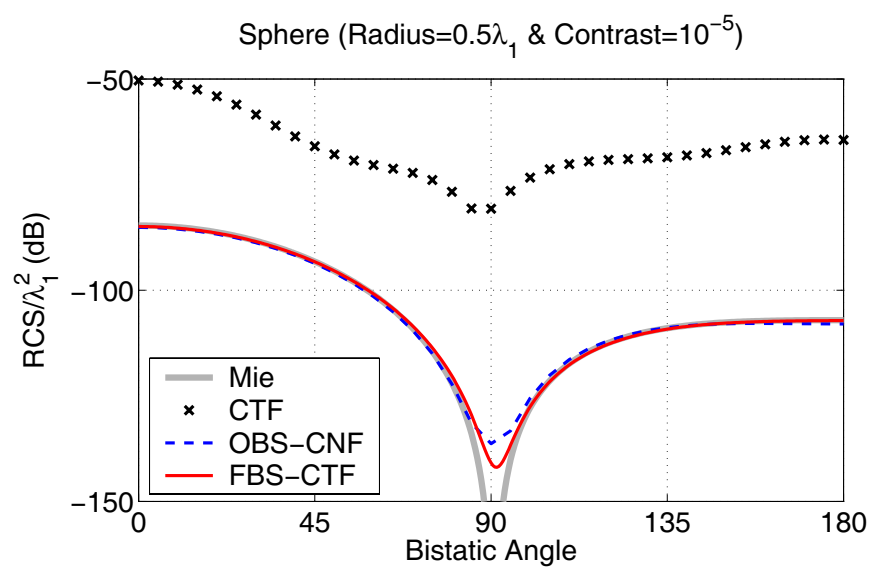

(b)

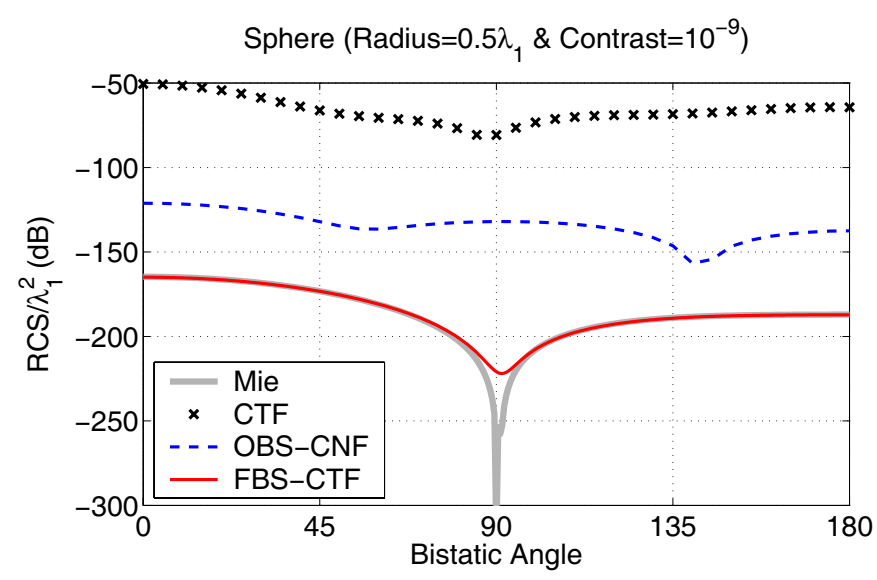

(c)

Fig. 3: Normalized bistatic $\operatorname{RCS}\left(\operatorname{RCS} / \lambda_{1}^{2}\right)$ of a sphere of radius $0.5 \lambda_{1}$ in free space, when the contrast is (a) $10^{-1}$, (b) $10^{-5}$, and (c) $10^{-9}$. In the plots, $0^{\circ}$ and $180^{\circ}$ correspond to forwardscattering and back-scattering directions, respectively.

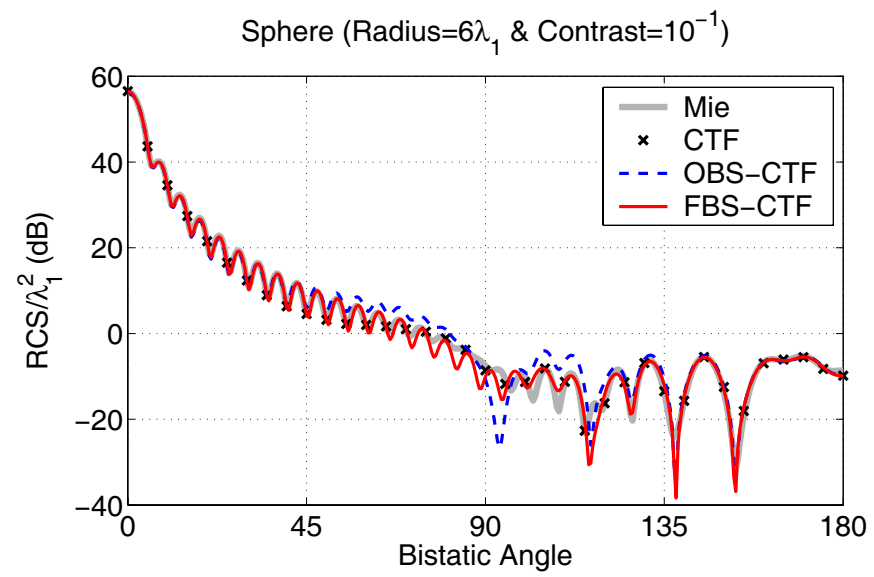

(a)

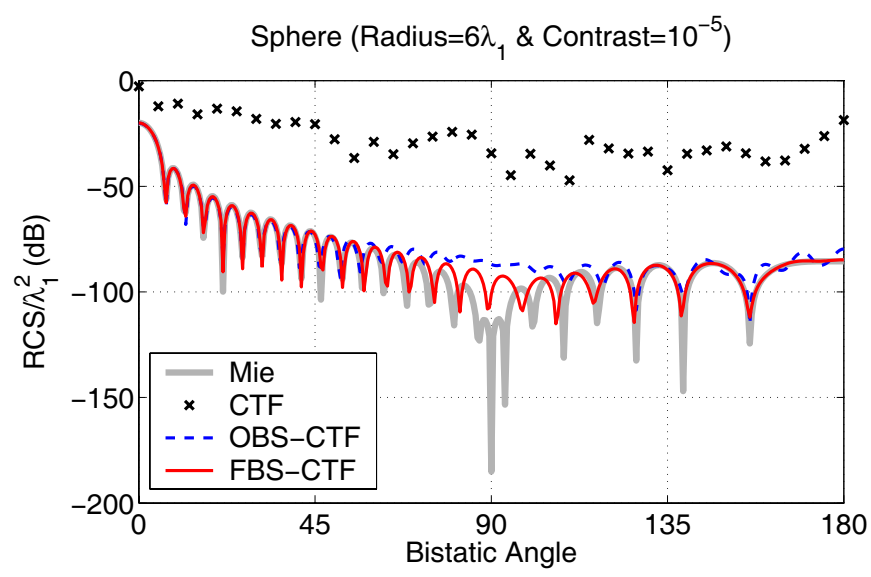

(b)

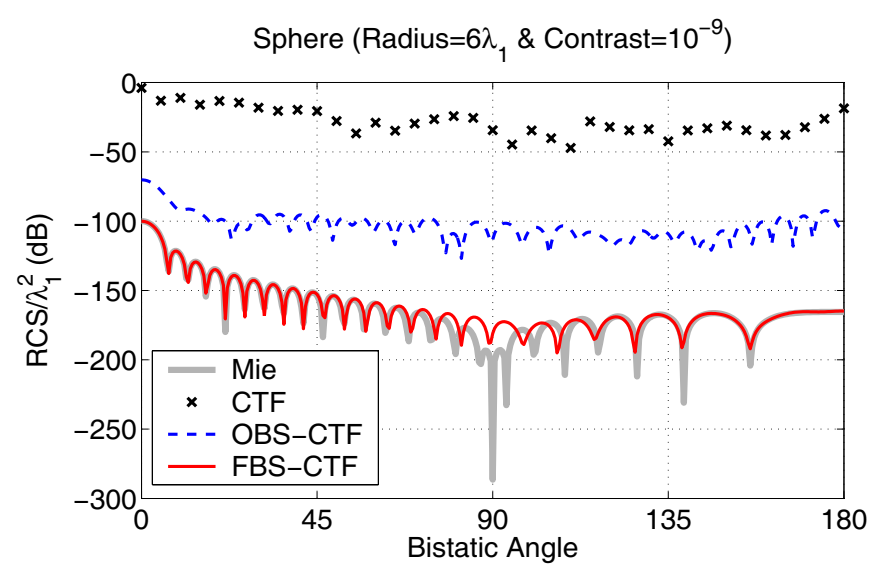

(c)

Fig. 4: Normalized bistatic RCS (RCS/ $\lambda_{1}^{2}$ ) of a sphere of radius $6 \lambda_{1}$ in free space, when the contrast is (a) $10^{-1}$, (b) $10^{-5}$, and (c) $10^{-9}$. In the plots, $0^{\circ}$ and $180^{\circ}$ correspond to forwardscattering and back-scattering directions, respectively. 